\title{
Modeling of Interfacial Modification Effects on Thermal Conductivity \\ of Carbon Nanotube Composites
}

\author{
Thomas C. Clancy \\ National Institute of Aerospace \\ 100 Exploration Way \\ Hampton, VA 23666 \\ Thomas S. Gates \\ Mechanics of Structures and Materials Branch \\ MS 188E \\ NASA Langley Research Center \\ Hampton, VA 23681
}

\begin{abstract}
:
The effect of functionalization of carbon nanotubes on the thermal conductivity of nanocomposites has been studied using a multi-scale modeling approach. These results predict that grafting linear hydrocarbon chains to the surface of a single wall carbon nanotube with covalent chemical bonds should result in a significant increase in the thermal conductivity of these nanocomposites. This is due to the decrease in the interfacial thermal (Kapitza) resistance between the single wall carbon nanotube and the surrounding polymer matrix upon chemical functionalization. The nanocomposites studied here consist of single wall carbon nanotubes in a bulk poly(ethylene vinyl acetate) matrix. The nanotubes are functionalized by end-grafting linear hydrocarbon chains of varying length to the surface of the nanotube. The effect which this functionalization has on the interfacial thermal resistance is studied by molecular dynamics simulation. Interfacial thermal resistance values are calculated for a range of chemical grafting densities and with several chain lengths. These results are subsequently used in an analytical model to predict the resulting effect on the bulk thermal conductivity of the nanocomposite.
\end{abstract}

\section{Introduction}

Polymers have many applications and uses but are often limited by their inherent physical properties. Most polymers have very low thermal conductivity. If the thermal conductivity could be improved without sacrificing the other properties of the polymer, more applications would be available for these materials [1]. A common commercial 
polymer with many uses is poly(ethylene vinyl acetate) (EVA), which has a relatively low thermal conductivity $(0.2 \mathrm{~W} / \mathrm{mK})$, typical for polymer materials. An approach of current interest to improve the thermal conductivity (TC) of these materials is the selective addition of nanostructured materials with high TC.

Although many novel nanostructured materials are available for study, the material chosen for this study is a single-walled carbon nanotube (SWCNT) composite. Carbon nanotubes (CNT) are known to have extremely high TCs [2] and research in measurement and modeling of carbon nanotube composite materials is currently very active. Thermal conductivity has been measured for a number of CNT polymer composites. However, to date, only modest increases in total TC have been measured experimentally [3]. At the nano-scale, it is assumed that there are a variety of intrinsic material structures which may be influential in establishing the properties of these nanocomposite systems. Previous theoretical work [4-6] has suggested that the modesty of these TC increases could be attributed primarily to the presence of a large interfacial thermal (Kapitza) resistance between the CNT and the surrounding polymer matrix. It is assumed that this interfacial resistance can be directly influenced by the degree of functionalization at the interface of the CNT and the polymer matrix. Recent simulation work has indicated the influence of interfacial molecular structure on interfacial thermal resistance [7].

The goal of the atomistic-based modeling presented herein is to address the issue of interfacial thermal resistance by exploring key structure-property relationships and establishing, through parametric studies, if increases in the TC of the composite can be achieved by proper functionalization of the CNT-polymer matrix interface. The 
interfacial thermal resistance, $R_{K}$, between the CNT and surrounding matrix is modeled with atomistic molecular dynamics (MD) simulations. Parameters associated with the functionalization of the CNT-EVA matrix interface are systematically varied. These parameters are the grafting density, $\sigma$, and length, $n$, of linear hydrocarbon chains covalently bonded to the CNT surface. The effects of defects in the CNT on the interfacial thermal resistance are also examined. Analytical modeling is also applied in order to consider the effect of various parameters on the TC of the composite. Key parameters to be varied in this analytical modeling include CNT length, CNT volume fraction, CNT thermal conductivity and interfacial thermal resistance.

\section{Method}

In the current work, computer simulations of SWCNTs in EVA have been performed. Atomistic structures with variations in CNT functionalization are first prepared using standard molecular dynamics (MD) techniques. A methodology [4-6] is applied to calculate the interfacial thermal resistance, $R_{K}$, in these structures. These $R_{K}$ values are then used with analytical modeling in order to consider the effect of chemical functionalization of SWCNTs on the thermal properties of the resulting nanocomposite.

\subsection{Structure preparation}

In order to predict the $R_{K}$ values between the SWCNT and the matrix, MD simulations were performed with short linear hydrocarbon chains $\left(-\mathrm{C}_{n} \mathrm{H}_{2 n+1}, n=8,12,18\right.$, 50) randomly end-grafted to the surface of a $(6,6)$ SWCNT. The SWCNT was

functionalized by this covalent end-grafting with a range of grafting densities ( $\sigma=0-0.11$ $\AA^{-2}$ ). Fig. 1 illustrates a typical SWCNT with end-grafted chains. The diameter of the SWCNT was approximately $1 \mathrm{~nm}$. These functionalized SWCNTs were embedded in an 
EVA matrix in the MD simulation. The amorphous EVA copolymer consisted of $15 \%$ vinyl acetate monomers by weight or 5.5\% mole fraction. In these simulations, 70 chains of EVA copolymer containing 25 repeat units each were simulated as a polymer matrix surrounding a $(6,6)$ SWCNT. The copolymer sequences for the EVA matrix were randomly constructed; however, this is relatively insignificant since each chain only contained 1-2 vinyl acetate monomers. The functionalized nanotubes were simulated in the bulk EVA matrix as a nanocomposite. Bulk density was effected through application of periodic boundary conditions ${ }^{8}$ in a simulation box size of about $46 \AA$ x $46 \AA$ x $58 \AA$. The SWCNT was extended through the $z$ (axial) coordinate of length $58 \AA$. In all the simulations, the PCFF force field ${ }^{9}$ was used with the LAMMPS $[10,11]$ MD simulation software. The SWCNT atoms are not constrained in any way other than that necessitated by the periodic boundary conditions.

A total of 17 structures were constructed and thermally equilibrated. These included one structure with no functionalization of the SWCNT and 13 structures with variable length, $n$, and grafting density, $\sigma$, of end-grafted chains. Three more structures were variations involving defects of the nanotube. The largest range of grafting densities was constructed with the 8 carbon chain $\left(-\mathrm{C}_{n} \mathrm{H}_{2 n+1}, n=8\right)$ end-grafts, where the SWCNTs with the following four grafting densities were simulated in an EVA matrix: $(\sigma=0.0068$, 0.013, $\left.0.054,0.11 \AA^{-2}\right)$. The SWCNTs with 12 carbon chain end-grafts $(n=12)$ were simulated at grafting densities: $\left(\sigma=0.0034,0.0068,0.013,0.027,0.054 \AA^{-2}\right)$. The SWCNTs with 18 carbon chain end-grafts $(n=18)$ were simulated at grafting densities: $\left(\sigma=0.0068,0.013 \AA^{-2}\right)$, and the SWCNTs with 50 carbon chain end-grafts $(n=50)$ were simulated at grafting densities: $\left(\sigma=0.0034,0.0068 \AA^{-2}\right)$. 
The bulk atomistic nanocomposite model is constructed by compression from low density. This procedure begins by placing the SWCNT with grafted chains and the 70 polymer molecules in a box with periodic boundary conditions. This initial density is about $5 \%$ of the bulk density of $0.8 \mathrm{~g} / \mathrm{cm}^{3}$. To prevent the polymer chains from collapsing, the Van der Waals (VDW) potential was scaled by a factor of 0.001 and a short cutoff (3.0 §) was applied with Coulomb interactions turned off. To allow the polymer chains to diffuse through the periodic box and achieve random configurations, the MD simulations were run for 50 ps using a 1 fs timestep at $500 \mathrm{~K}$, reducing the volume at a linear rate to a factor of about $10 \%$ of the final bulk density. A second MD run condensed the system to a density of about $50 \%$ of the final bulk density over 50 ps at $500 \mathrm{~K}$. The unscaled VDW potential is then applied with a cutoff of $9.0 \AA$ and Coulomb interactions are enacted with the Ewald summation technique. Energy minimization is performed on the system followed by constant pressure MD simulation. MD simulation is applied for $10 \mathrm{ps}$ while increasing the pressure from 1 to $100 \mathrm{atms}$ at $300 \mathrm{~K}$. This is followed by a compression at $100 \mathrm{atms}$ for $50 \mathrm{ps}$ at $300 \mathrm{~K}$. The pressure is reduced from 100 to 1 atms over $10 \mathrm{ps}$. This is followed by a MD run at $1 \mathrm{~atm}$ for $100 \mathrm{ps}$ and $300 \mathrm{~K}$. 2.2. Simulation of the heat transfer

Following the last MD run of the atomistic structures at $300 \mathrm{~K}$ described in Section 2.1, a methodology [4-6] was applied to obtain the interfacial thermal resistance, $R_{K}$. The interfacial thermal resistance calculation involved the instantaneous heating of the SWCNT atoms to a temperature of $500 \mathrm{~K}$. Following this instantaneous heating, the simulation was run at constant energy, as thermal energy was transferred from the SWCNT to the surrounding polymer matrix. The difference in temperature, $\Delta T$, between 
the SWCNT atoms and the other atoms was recorded as a function of MD timestep. Fig. 2 shows a plot of the $\ln (\Delta T)$ as a function of time since the application of the instantaneous heating. A least squares fit to this curve yields a slope which is the negative inverse of the characteristic decay time, $\tau$. The interfacial thermal resistance, $R_{K}$, [6] is calculated from Eq. 1, where $c_{T} / A_{T}$ is the heat capacity per area of SWCNT surface (using a value of $5.6 \times 10^{-4} \mathrm{~J} / \mathrm{m}^{2} \mathrm{~K}$ for $c_{T} / A_{T}[4]$.)

$$
R_{K}=\frac{\tau}{\left(c_{T} / A_{T}\right)}
$$

\subsection{Analytical modeling of the thermal conductivity}

These interfacial resistance values from the heat transfer simulations are then used in an analytical formula [12] to calculate the TCs of nanocomposites. While molecular modeling yields $R_{K}$ values, an analytical model is applied in order to calculate the thermal conductivity of the nanocomposite material. This model assumes a random orientation of the dispersed nanotubes within the polymer matrix and uses an effective medium approach. This approach yields a formula for calculating the TC of carbon nanotube composites [12]. This is summarized by Eqs. 2-5.

$$
\begin{gathered}
K_{e}=K_{m} \frac{3+f\left(\beta_{x}+\beta_{z}\right)}{2-f \beta_{x}} \\
\beta_{x}=\frac{2\left(K_{11}^{c}-K_{m}\right)}{K_{11}^{c}+K_{m}}, \quad \beta_{z}=K_{33}^{c} / K_{m}-1 \\
K_{11}^{c}=\frac{K_{c}}{1+\frac{2 a_{k}}{d} \frac{K_{c}}{K_{m}}}, \quad K_{33}^{c}=\frac{K_{c}}{1+\frac{2 a_{k}}{L} \frac{K_{c}}{K_{m}}} \\
a_{K}=R_{K} K_{m}
\end{gathered}
$$


Material properties $K_{e}, K_{c}$, and $K_{m}$ are the thermal conductivities of the nanocomposite, the CNT and the polymer matrix, respectively. In evaluating Eqs. 2-5, a value of 0.2 $\mathrm{W} / \mathrm{mK}$ is used for $K_{m}$. Two values are used for $K_{c}: 6000 \mathrm{~W} / \mathrm{mK}$ and $3000 \mathrm{~W} / \mathrm{mK}$ [2, 5]. The diameter of the $(6,6)$ SWCNT, $d$, is $8.136 \AA$, while $f$ is the volume fraction, $L$ is the nanotube length and $a_{K}$ is the Kapitza radius.

\section{Results and Discussion}

This section will describe the results of the modeling and provide discussion on the observed behaviors. In Section 3.1 the prepared atomistic structures are examined. In Section 3.2 the results of the MD heat transfer are discussed. From these simulations, the interfacial thermal resistant values, $R_{K}$, for a range of functionalization conditions are calculated. In Section 3.3, these $R_{K}$ values are then used with analytical modeling $[12,13]$ to predict the TC of the composites. By systematically varying the CNT length, volume fraction, thermal conductivity and the interfacial thermal resistance, key structureproperty relationships were established and related directly to changes in the predicted TC of the composite.

3.1. Radial density profiles of the prepared structures

The radial density profiles of the nanocomposites are examined in order to determine if there are large differences in mass distribution, particularly near the CNTmatrix interface which may be significant. Fig. 3a shows the radial distribution functions of the mass density as a function of distance, $r$, about the center of the SWCNT. These profiles are shown for the MD simulations with $n=12$ linear hydrocarbon end-grafted chains. The large peak at $\sim 5 \AA$ is due to the CNT wall. At low to moderate grafting 
densities, $\left(\sigma=0-0.027 \AA^{-2}\right.$ ), the density profiles show the familiar oscillating behavior associated with packing polymer chains at surfaces. At high grafting density $(\sigma=0.054$ $\AA^{-2}$ ), the profile has a qualitative change in shape, due to the exclusion of polymer matrix chains from the SWCNT surface. Fig. 3b shows the same profiles given only by the contribution from the SWCNT and the end-grafted chains. A qualitative change in the profile shape occurs at high grafting density $\left(\sigma=0.054 \AA^{-2}\right)$ due to the steric crowding of the grafted chains.

\subsection{MD heat transfer results}

The results obtained from the MD simulation described in Section 2.2 are presented here. The 17 separate structures representing the variation in grafting density and end-grafted chain length are each subjected to the methodology involving instantaneous heating of the SWCNT atoms. Decay plots similar to Fig. 2 are obtained for each structure and the interfacial resistance values, $R_{K}$, are calculated according to Eq. 1. Fig. 4a shows a plot of $R_{K}$ as a function of grafting density for the various end-grafted chain lengths $\left(-\mathrm{C}_{n} \mathrm{H}_{2 n+1}, n=8,12,18,50\right)$. Large decreases in $R_{K}(>80 \%)$ are observed with increasing grafting density for all chain lengths. A consistent correlation between chain length and decreasing $R_{K}$ is predicted for corresponding grafting densities.

The grafting considered above assumes a pristine SWCNT with no defects and grafting taking place at sp3 carbons located adjacent to the same locations as the previously unfunctionalized sp2 carbon atoms, i.e. direct covalent bonding to a pristine nanotube. However, it is reasonably assumed that during synthesis bonding could occur at defect regions in the nanotube wall. To consider this type of structure, the bonding is altered in several cases to approximate the effect of defects adjacent to bonding sites. An 
sp3 carbon is bonded to 4 atoms. In the case of the grafted chains, the sp3 carbon of the nanotube is bonded to the terminal carbon atom of the linear hydrocarbon and 3 other carbon atoms in the CNT. In order to approximate the effect of a defect, 2 of these sp3sp2 carbon-carbon bonds are broken. This does not result in the removal of any atoms, although the bonding characteristics of atoms adjacent to the grafting point are substantially altered. This is illustrated in Fig. 4b, where a planar graphite section representing the CNT wall is shown. A hydrocarbon chain is bonded to the CNT wall.

With these defects in place, the simulation is repeated for the $n=12$ chains for several grafting densities. Results, presented in Fig. 4c, compare the interfacial thermal resistance of the pristine grafted nanotube with the resistance of the defect induced nanotube. Very little difference in interfacial thermal resistance is predicted between the two cases, despite the very different bonding characteristics in the vicinity of the grafted point. Specifically, in the defect induced nanotube, the polymer chain is essentially endgrafted to the edge of a hole in the nanotube. The results indicate that for the materials of this study, SWCNT defects will not have a major effect on the interfacial thermal resistance as influenced by the grafting of chains.

The results of the heat transfer simulations are summarized in Table 1 . The range of grafting densities, $\sigma$, is indicated by the columns. The range of end-grafted chain lengths, $n$, is indicated by the rows. The interfacial thermal resistance values are placed in this array in units of $\mathrm{m}^{2} \mathrm{~K} / \mathrm{W}\left(\mathrm{x} 10^{8}\right)$. The values in parentheses are for the simulations with the defects in the SWCNTs. Comparing grafting densities in terms of percentage of functionalized carbons, there are 576 carbon atoms in the SWCNT. By percentage of functionalized carbon atoms in the CNT, the grafting densities are: $(0.86 \%, 1.74 \%$, 
3.47\%, 6.9\%, and 13.9\%) corresponding to grafting densities $(\sigma=0.0034,0.0068,0.013$, 0.027, 0.054, and $0.108 \AA^{-2}$.)

\subsection{Analytical modeling results}

Using the analytical modeling presented in Section 2.2 [12, 13], the thermal conductivity of the composite, $K_{e}\left(R_{K}\right)$ is plotted in Fig. 5 a. Here, a value of $1 \times 10^{-6} \mathrm{~m}(1$ micron) is used for the length, $L$ and $K_{c}=6000 \mathrm{~W} / \mathrm{mK}$. The volume fraction of nanotubes in the bulk material, $f$, ranges from $1-5 \%$ in these plots. This volume fraction is a parameter used in Eq. 2-5 and is not derived from any details of the MD simulations in Sections 3.1 and 3.2. The solid line is computed for a continuous range of values, while the symbols are marked for the specific $R_{K}$ values obtained from the $(n=8)$ MD simulations. These results illustrate the strong influence that $R_{K}$ has with this common nanotube length. Fig. 5b shows similar results plotted with a longer nanotube length $(L=$ 10 microns). Fig. 5c shows results plotted with a much longer nanotube length $(L=100$ microns). Comparing Figs. 5a-c, it is clear that the $R_{K}$ value is much more influential on TC at shorter CNT length. All three plots retain the same limiting values $\left(R_{K}=0\right)$, yet have strongly differing TC values at finite $R_{K}$. Fig. 6 shows the influence of NT length more directly. The TC of the nanocomposite is plotted as a function of NT length for several values of $R_{K}$. The five finite values of $R_{K}$ are taken from calculated values shown in Fig. 4a for $n=8$, and the limiting value $\left(R_{K}=0\right)$ is included. The plots clearly indicate the diminishing effect of CNT length with decreasing $R_{K}$.

Estimates for the thermal conductivity of CNTs vary considerably [2, 14-17]. The effect of functionalization may decrease the thermal conductivity [17]. We have arbitrarily used a fixed value of $6000 \mathrm{~W} / \mathrm{mK}$ for the calculations presented in Figs. 5a-c 
in order to focus on the effect of $R_{K}$ on the TC of the nanocomposite. Fig. 7 illustrates the influence of the thermal conductivity of the CNT $\left(K_{c}\right)$, by recalculating the values from Fig. 5c with a change of $K_{c}$ from $6000 \mathrm{~W} / \mathrm{mK}$ to $3000 \mathrm{~W} / \mathrm{mK}$. This illustrates that a lower thermal conductivity of the nanotubes would still allow a significant improvement in the resulting TC of the nanocomposite.

Fig. 8 shows the results from Fig. 5c as a function of grafting density, $\sigma$. This more clearly illustrates the dependency of the resulting TC of the nanocomposite on the grafting density of chains $(n=8)$ attached to the CNT. The dependence of the thermal conductivity on the chain length, $n$, is shown in Fig. 9. The grafting density for the data in this plot is $\sigma=0.0068 \AA^{-2}$, while the other values are the same as in Fig. 5c.

\section{Summary}

Computer simulations of CNTs in an EVA matrix were performed in order to evaluate the effect of various parameters on thermal conductivity of the composite. In the atomistic-based modeling, interfacial thermal resistance values, $R_{K}$, were calculated as a function of several functionalization parameters. The values were found to be strongly influenced by the grafting density, $\sigma$, and chain length, $n$, of short linear hydrocarbon chains covalently bonded to the CNT surface. Increasing chain length and grafting density lowers the interfacial thermal resistance. The presence of defects adjacent to grafting sites causes very little change in the predicted values of $R_{K}$.

In the analytical modeling, the TC of the composite was found to be strongly dependent on CNT length, CNT volume fraction, CNT thermal conductivity, and interfacial thermal resistance. The dependence of TC on $R_{K}$ was most pronounced at shorter CNT lengths. CNT length was a critical factor in the resulting TC of composites 
at finite values of $R_{K}$. The TC increased with increasing $\sigma$ and $n$, but did not exhibit a

linear dependence.

\section{Acknowledgements}

The strategy for grafting linear chains was considered according to experimental work of

Dr. Joseph G. Smith of NASA-LaRC.

\section{References}

1. $\quad$ King, J. A.; Weber, E. H.; C., Q.; Tucker, K. W.; Vogt, B. D., Conductive High Temperature Nylon. Journal of Composite Materials 2000, 34, (24), 2038-2060.

2. $\quad$ Berber, S.; Kwon, Y.-K.; Tománek, D., Unusually High Thermal Conductivity of Carbon Nanotubes. Phys. Rev. Lett. 2000, 84, 4613.

3. Biercuk, M. J.; Llaguno, M. C.; Radosavljevic, M.; Hyun, J. K.; Johnson, A. T.; Fischer, J. E., Carbon nanotube composites for thermal management. Applied Physics Letters 2001, 80, (15), 2767-2769.

4. $\quad$ Huxtable, S.; Cahill, D.; Shenogin, S.; Xue, L.; Ozisik, R.; Barone, P.; Usrey, M.; Strano, M.; Siddons, G.; Shim, M.; Keblinski, P., Interfacial heat flow in carbon nanotube suspensions. Nature Materials 2003, 2, 731-734.

5. $\quad$ Shenogin, S.; Bodapati, A.; Xue, L.; Ozisik, R.; Keblinski, P., Effect of chemical functionalization on thermal transport of carbon nanotube composites. Applied Physics Letters 2004, 85, (12), 2229-2231.

6. $\quad$ Shenogin, S.; Xue, L.; Ozisik, R.; Keblinski, P., Role of thermal boundary resistance on the heat flow in carbon-nanotube composites. Journal of Applied Physics 2004, 95, (12), 8136-8144.

7. Patel, H. A.; Garde, S.; Keblinski, P., Thermal Resistance of Nanoscopic LiquidLiquid Interfaces: Dependence on Chemistry and Molecular Architecture. Nanoletters 2005, 5, (11), 2225-2231.

8. $\quad$ Allen, M. P.; Tildesley, D. J., Computer Simulation of Liquids. ed.; Oxford University Press: Oxford, 1987; 'Vol.' p.

9. $\quad$ Sun, H.; Mumby, S. J.; Maple, J. R.; Hagler, A. T., An ab Initio CFF93 All-Atom Force Field for Polycarbonates. Journal of the American Chemical Society 1994, 116, (7), 2978-2987.

10. http://www.cs.sandia.gov/ sjplimp/lammps.html

11. Plimpton, S. J., Fast Parallel Algorithms for Short-Range Molecular Dynamics. J. Comp. Phys. 1995, 117, 1-19.

12. Nan, C.; Liu, G.; Lin, Y.; Li, M., Interface effect on thermal conductivity of carbon nanotube composites. Applied Physics Letters 2004, 85, (16), 3549-3551.

13. Nan, C.; Birringer, R.; Clark, D. R.; Gleiter, H., Effective thermal conductivity of particulate composites with interfacial thermal resistance. Journal of Applied Physics 1997, 81, (10), 6692-6699. 
14. Ruoff, R. S.; Lorents, D. C., Mechanical And Thermal Properties of Carbon Nanotubes. Carbon 1995, 33, (7), 925-930.

15. Che, J.; Cagin, T.; Goddard, W. A., Thermal conductivity of carbon nanotubes. Nanotechnology 2000, 11, 65-69.

16. Osman, M. A.; Srivastava, D., Temperature dependence of the thermal conductivity of single-wall carbon nanotubes. Nanotechnology 2001, 12, 21-24.

17. Padgett, C. W.; Brenner, D. W., Influence of Chemisorption on the Thermal Conductivity of Single-WallCarbon Nanotubes. Nanoletters 2004, 4, (6), 1051-1053. 
Table 1 Interfacial thermal resistance values from Figs. $4 \mathrm{a}$ and $4 \mathrm{~b}$ in units of $\mathrm{m}^{2} \mathrm{~K} / \mathrm{W}$ $\left(\times 10^{8}\right)$. The grafting density is shown across the top row. The length of the grafted linear hydrocarbon is shown across the left column. The values for Fig. $4 \mathrm{~b}$ with the defects simulation are shown in parentheses.

\begin{tabular}{|c|c|c|c|c|c|c|c|c|}
\hline \multicolumn{9}{|c|}{$\sigma$} \\
\hline \multirow{6}{*}{$n$} & & 0 & 0.003 & 0.007 & 0.014 & 0.027 & 0.054 & 0.108 \\
\hline & 0 & 9.6 & & & & & & \\
\hline & 8 & & & 4.5 & 2.5 & & 0.6 & 0.2 \\
\hline & 12 & & $5.2(6.1)$ & $3.2(3.5)$ & 2.2 & $1.2(1.8)$ & 0.5 & \\
\hline & 18 & & & 2.6 & 1.5 & & & \\
\hline & 50 & & 4.8 & 1.9 & & & & \\
\hline
\end{tabular}




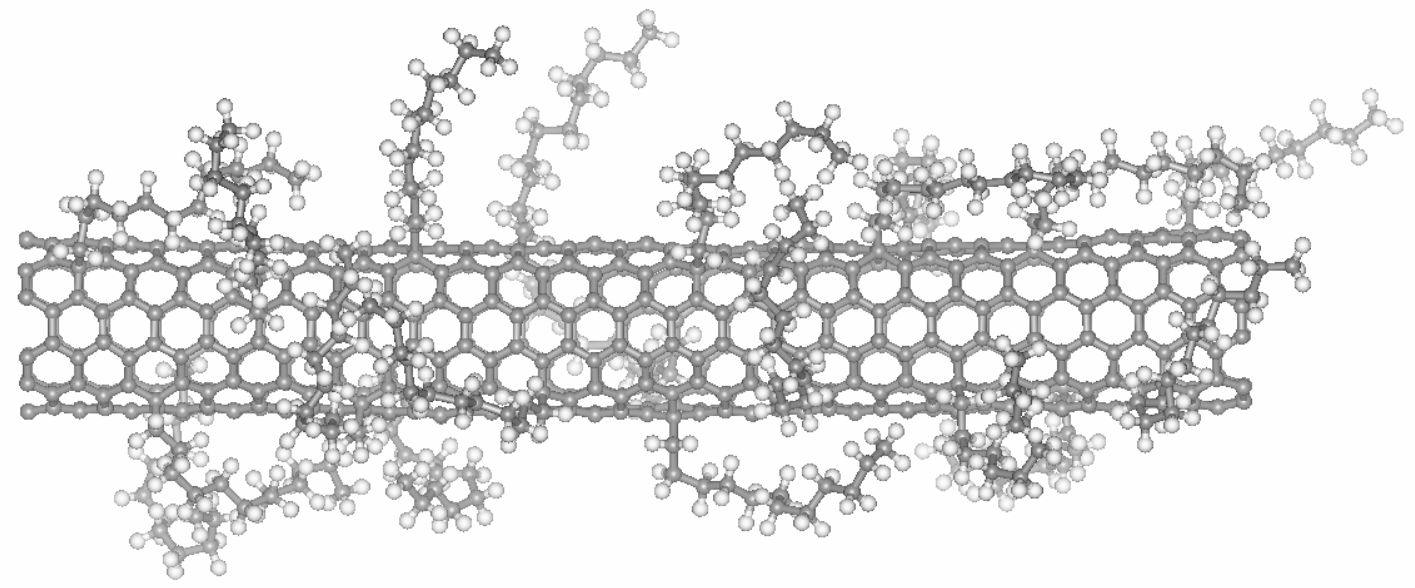

Grafting Density of $0.01352 \AA^{-2}, 20$ chains, $(6,6)$ SWCNT

Fig. 1. A single walled carbon nanotube (SWCNT) with short end-grafted chains is depicted. 


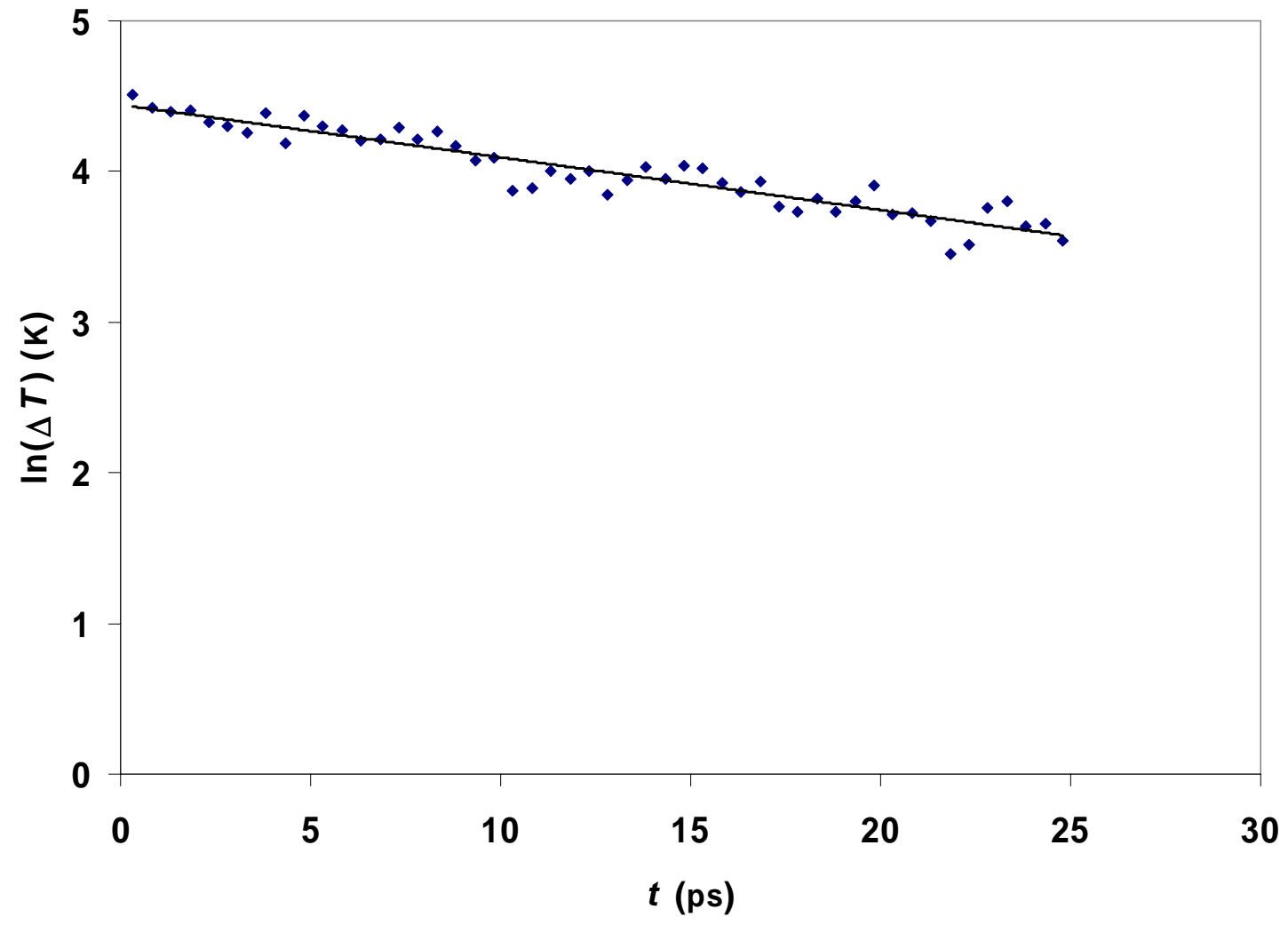

Fig. 2. The decay of the temperature difference between the carbon nanotube (CNT) and the polymer matrix. 


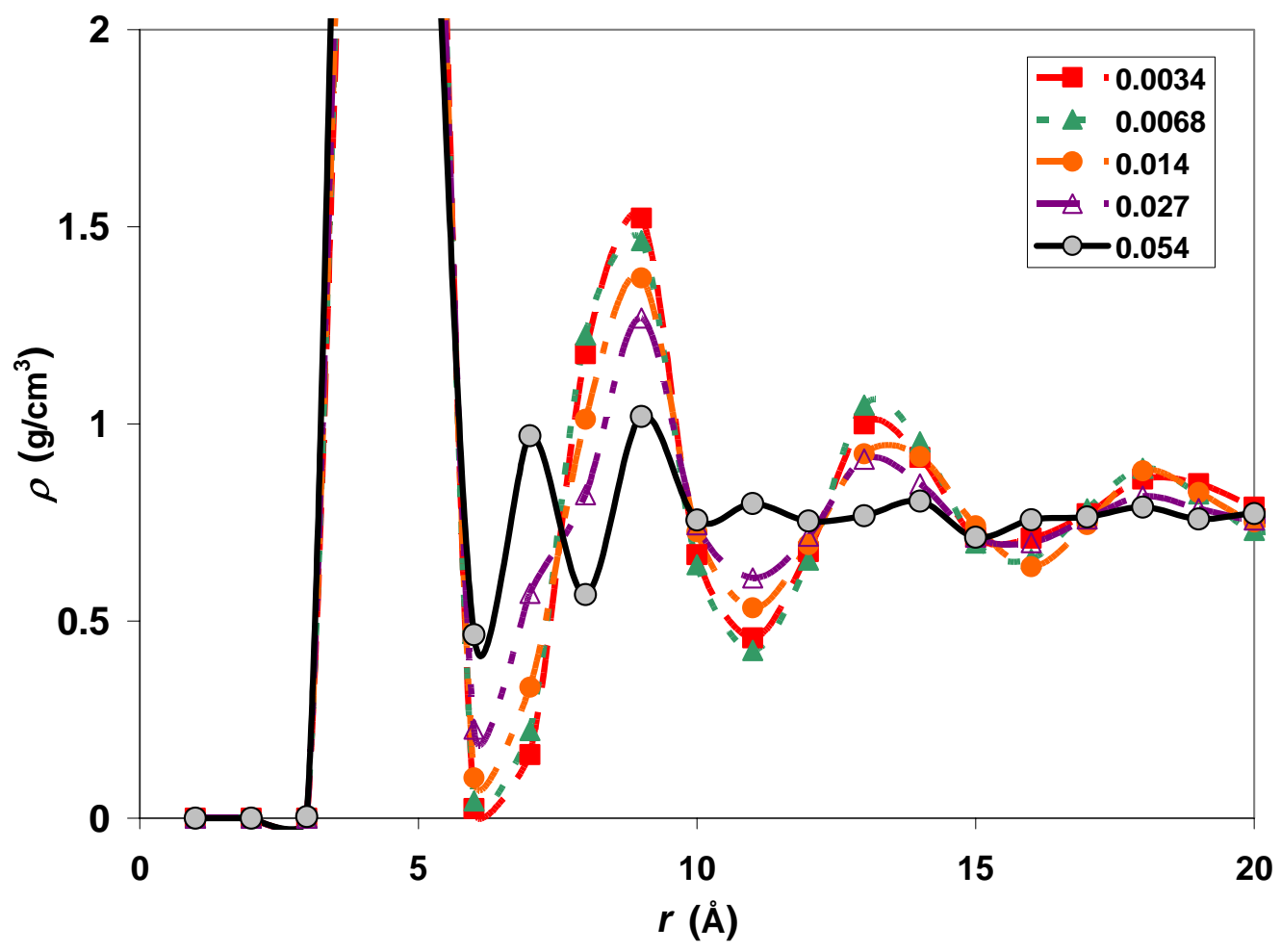

Fig. 3a. The radial density profiles for the nanocomposite system with $\mathrm{C}_{12} \mathrm{H}_{25}$ chains end-grafted to the nanotube in a poly(ethylene vinyl acetate) matrix for a range of grafting densities. The large peak at $\sim 5 \AA$ is due to the carbon nanotube wall. At high grafting density $\left(\sigma=0.054 \AA^{-2}\right)$, the profile has a qualitative change in shape. 


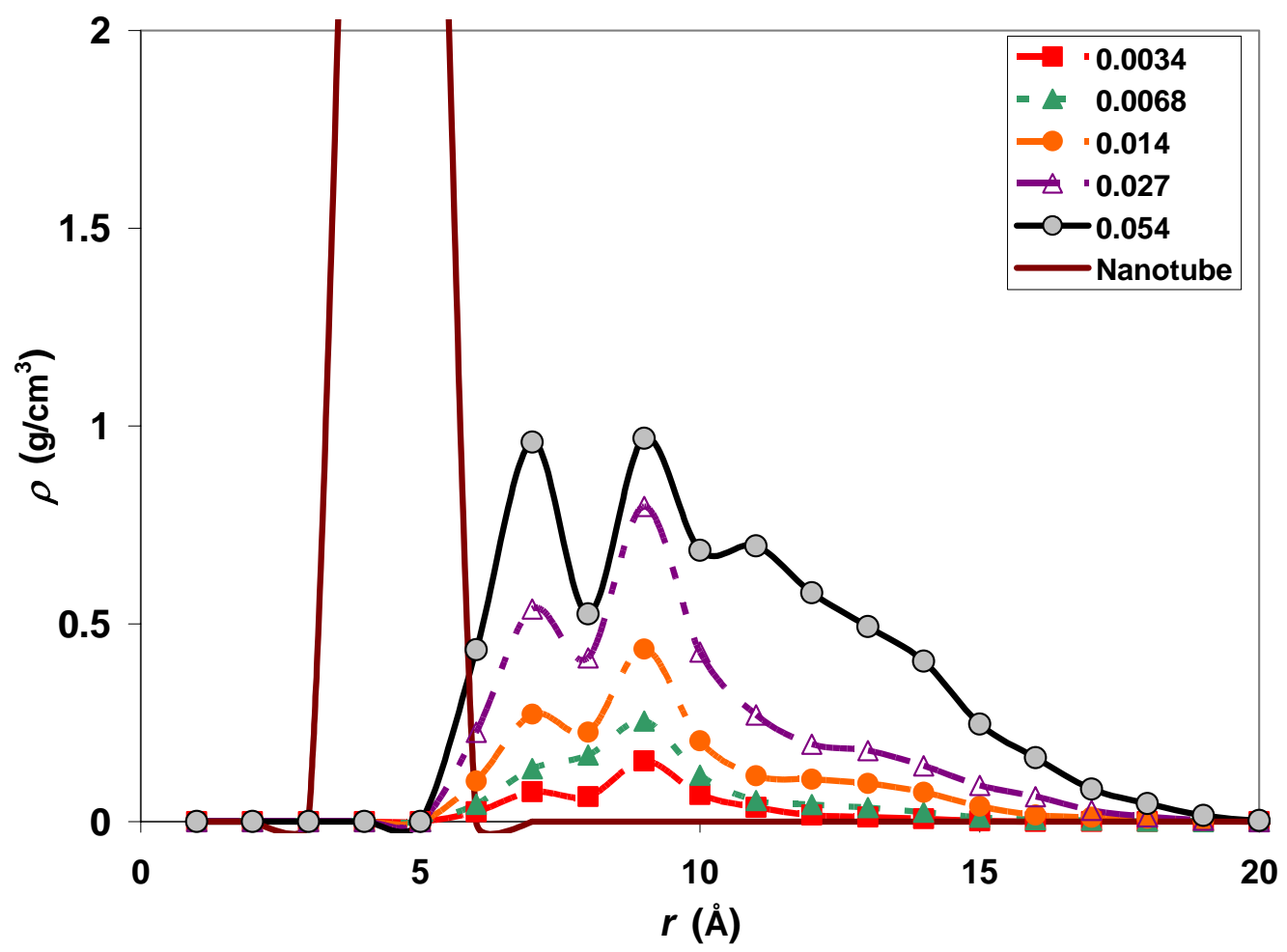

Fig. 3b. The radial density profiles for the nanocomposite system with $\mathrm{C}_{12} \mathrm{H}_{25}$ chains end-grafted to the nanotube in a poly(ethylene vinyl acetate) matrix for a range of grafting densities. The large peak at $\sim 5 \AA$ is due to the carbon nanotube wall. The density profiles are due to the end-grafted chains. The density profile contribution from the matrix has been removed. A qualitative change in the profile occurs at high grafting density $\left(\sigma=0.054 \AA^{-2}\right)$, as the chains become extended due to crowding. 


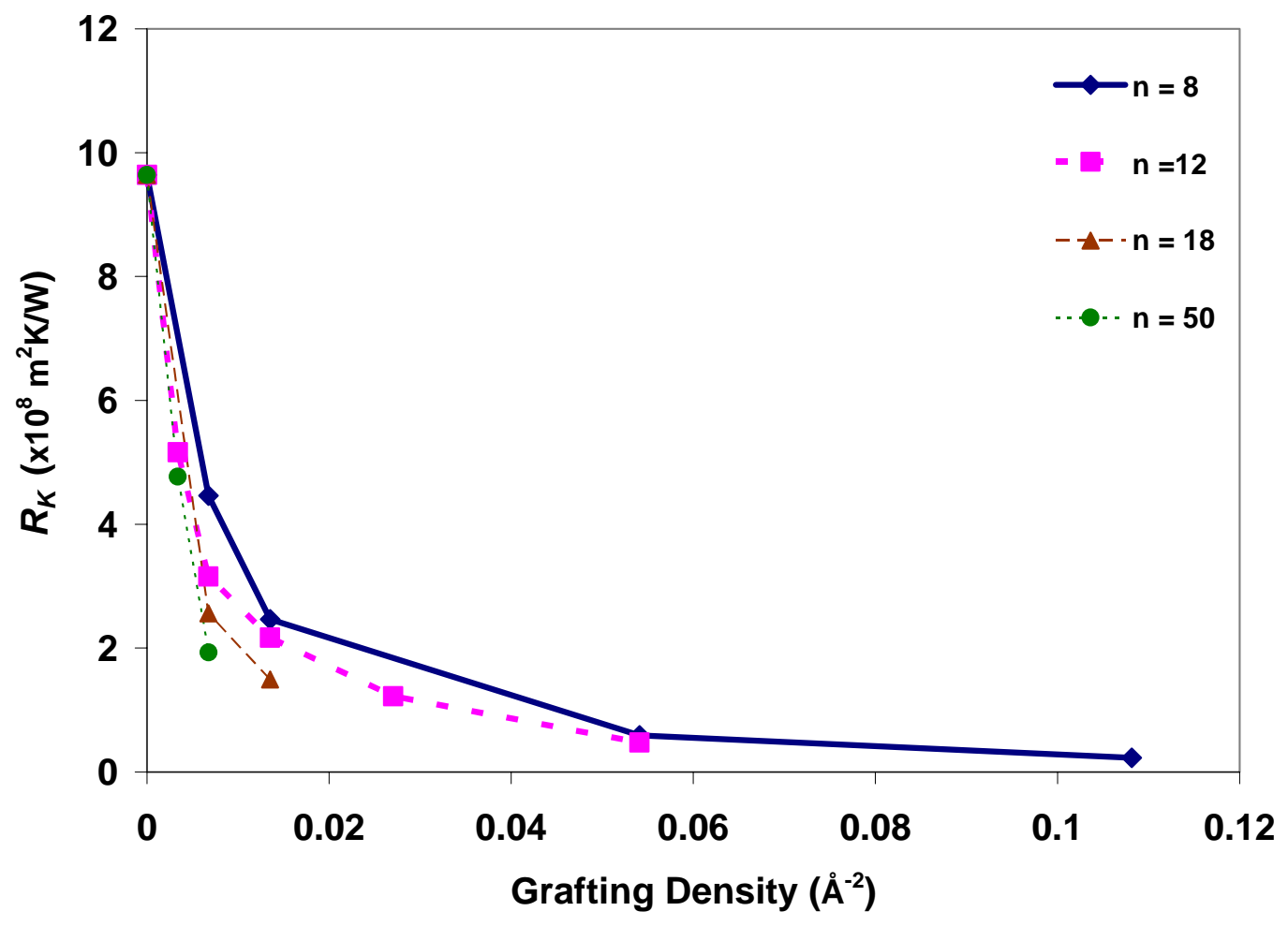

Fig. 4a. The interfacial thermal resistance $\left(R_{K}\right)$ is plotted as a function of the grafting density, $\sigma$, for several end-grafted chain lengths. 


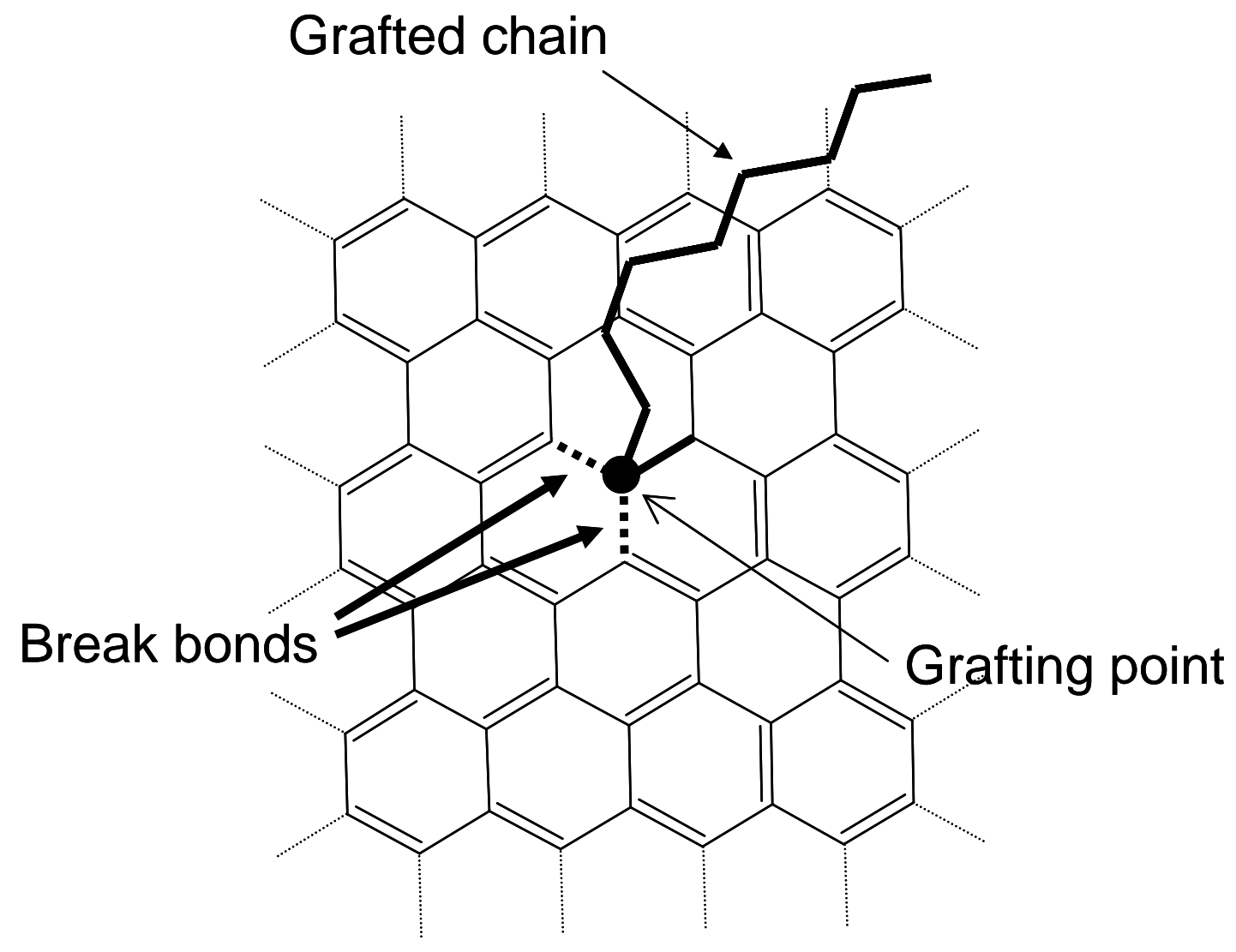

Fig. 4b. Defects are approximated by breaking two of the bonds adjacent to a grafting point. 


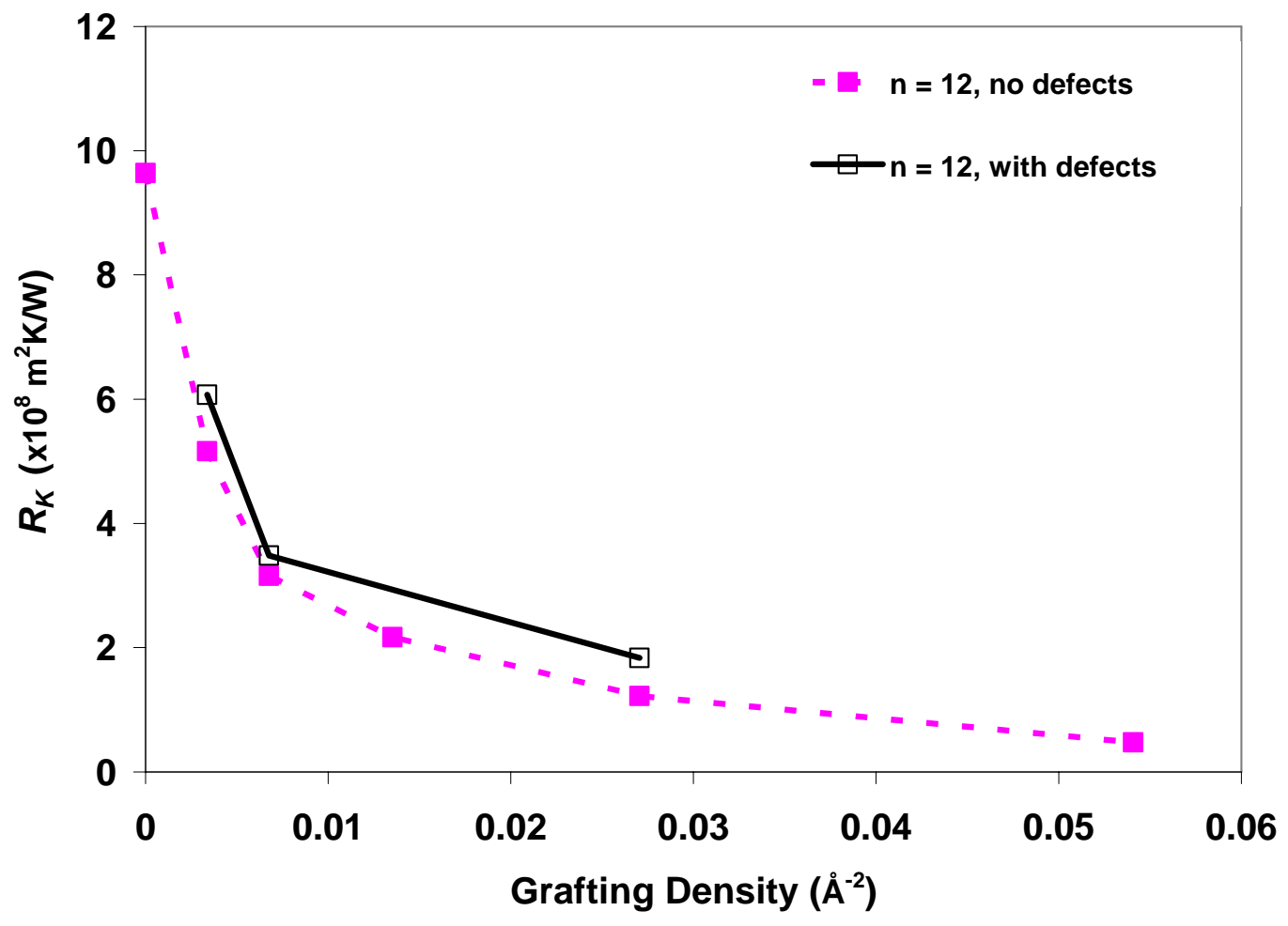

Fig. 4c. The interfacial thermal resistance $\left(R_{K}\right)$ is plotted as a function of the grafting density, $\sigma$, for pristine nanotubes and nanotubes with defects. Results are shown from MD simulations with grafted chain length, $n=12$. 


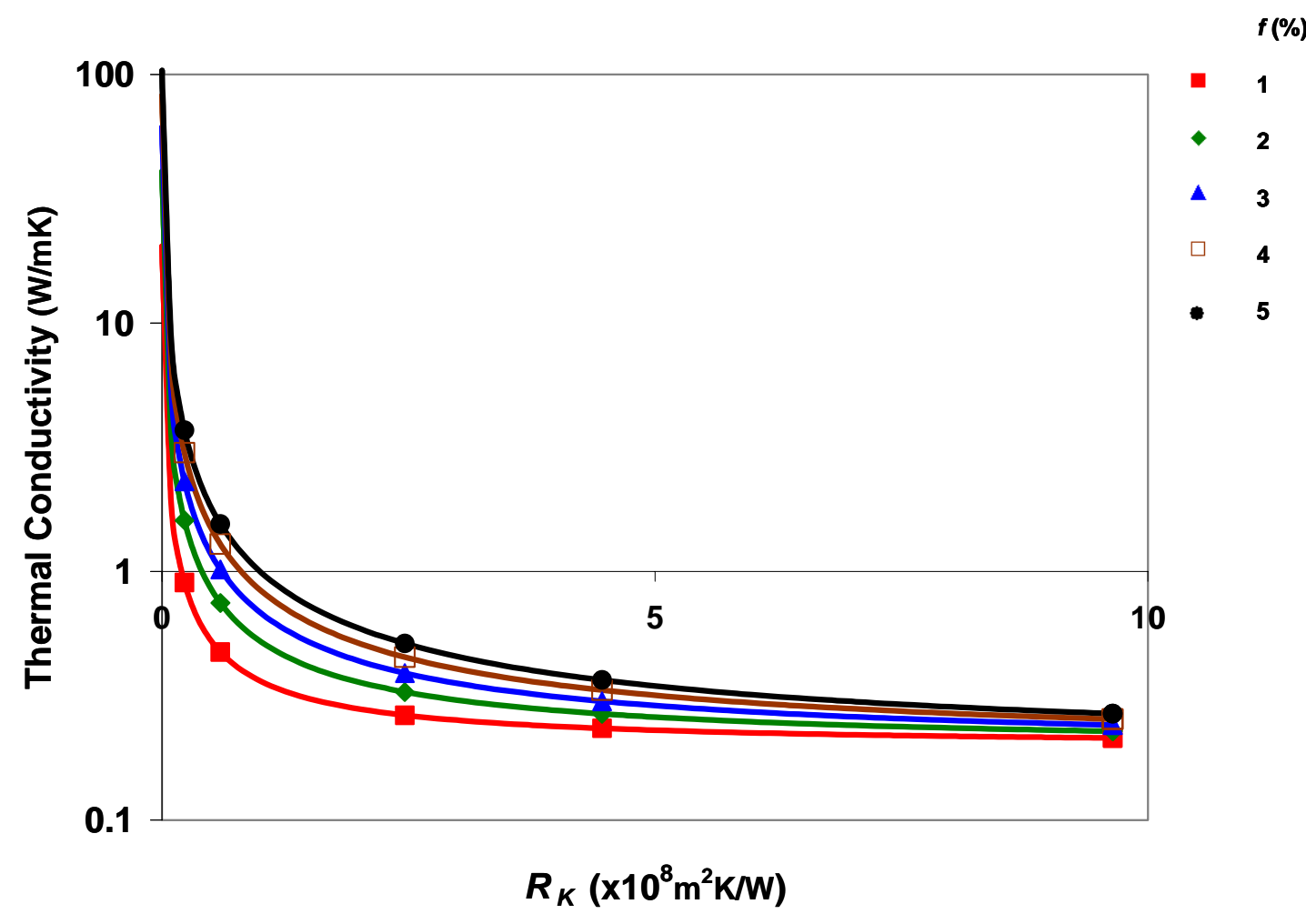

Fig. 5a. The thermal conductivity of the nanocomposite as a function of interfacial thermal resistance $\left(R_{K}\right)$ for a range of volume fractions $(f=1-5 \%)$. A value of $10^{-6} \mathrm{~m}(1$ micron) is used for the nanotube length, $L$. A value of $6000 \mathrm{~W} / \mathrm{mK}$ is used for the conductivity of the nanotube, $K_{c}$. The plotted points correspond to values obtained form the MD simulation for end-grafted chains of length $n=8$. 


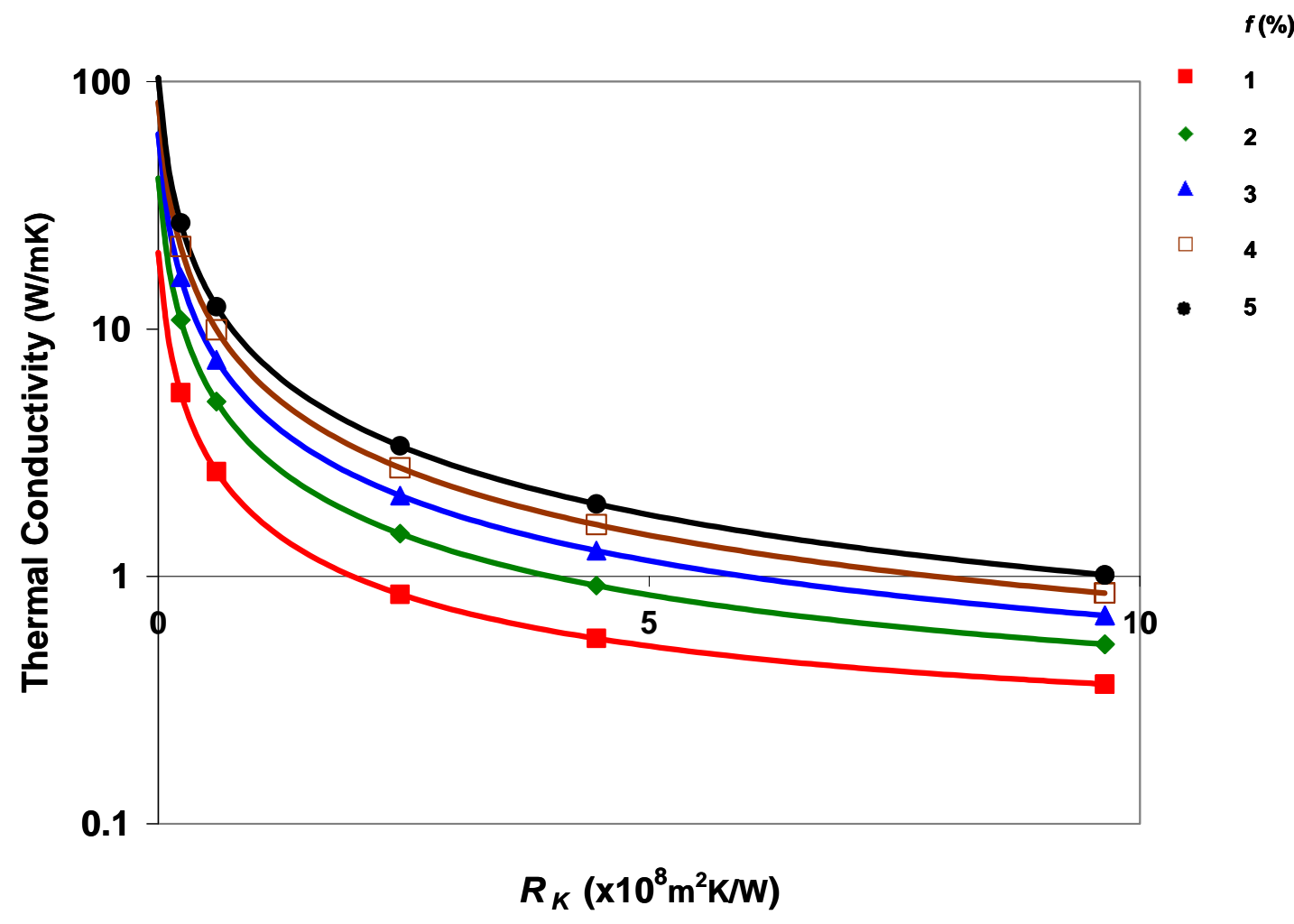

Fig. 5b. The thermal conductivity of the nanocomposite as a function of interfacial thermal resistance $\left(R_{K}\right)$ for a range of volume fractions $(f=1-5 \%)$. A value of $10^{-5} \mathrm{~m}(10$ microns) is used for the nanotube length, $L$. A value of $6000 \mathrm{~W} / \mathrm{mK}$ is used for the conductivity of the nanotube, $K_{c}$. The plotted points correspond to values obtained form the MD simulation with end-grafted chains of length $n=8$. 


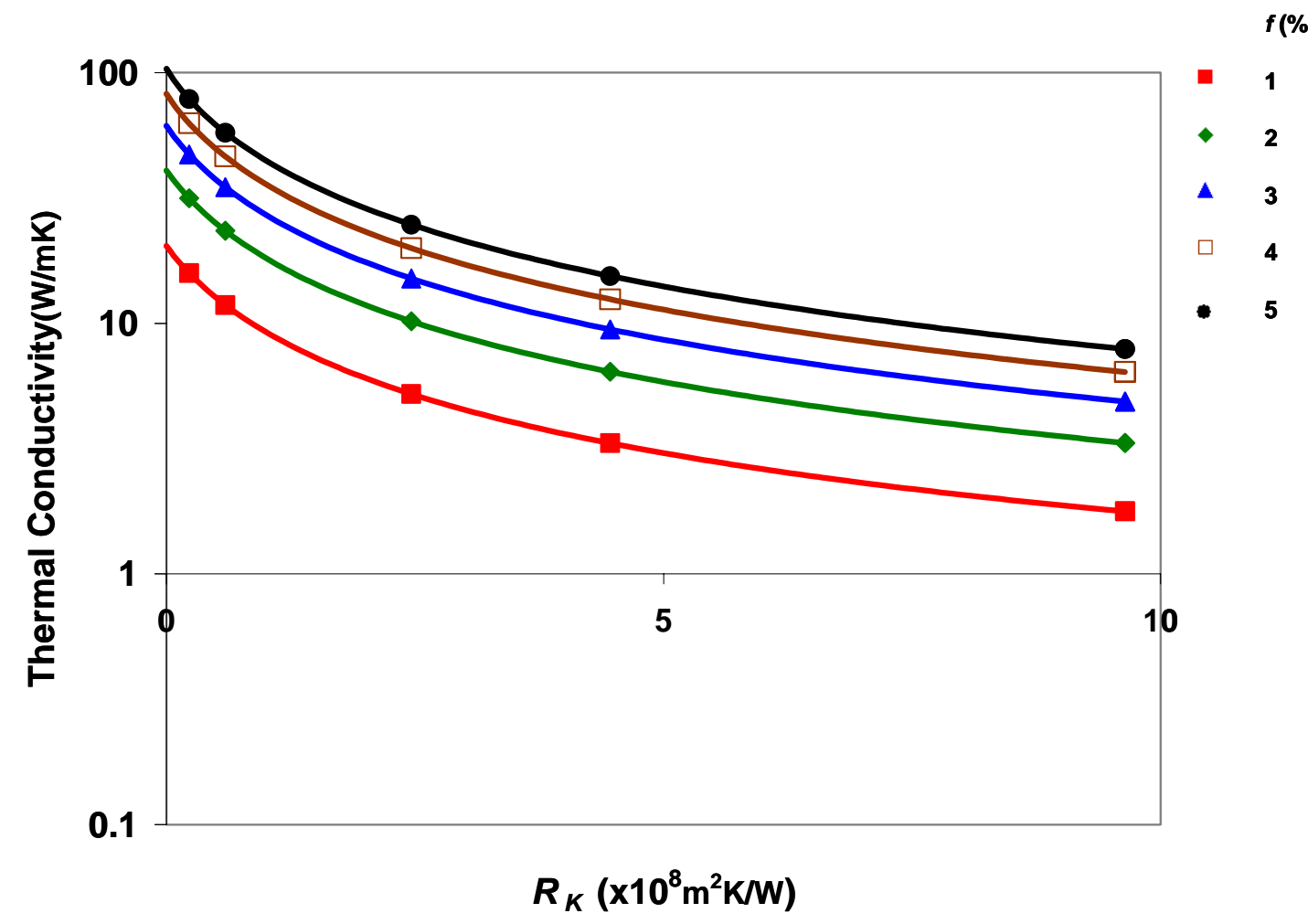

Fig. 5c. The thermal conductivity of the nanocomposite as a function of interfacial thermal resistance $\left(R_{K}\right)$ for a range of volume fractions $(f=1-5 \%)$. A value of $10^{-4} \mathrm{~m}$ (100 microns) is used for the nanotube length, $L$. A value of $6000 \mathrm{~W} / \mathrm{mK}$ is used for the conductivity of the nanotube, $K_{c}$. The plotted points correspond to values obtained form the MD simulation with end-grafted chains of length $n=8$. 


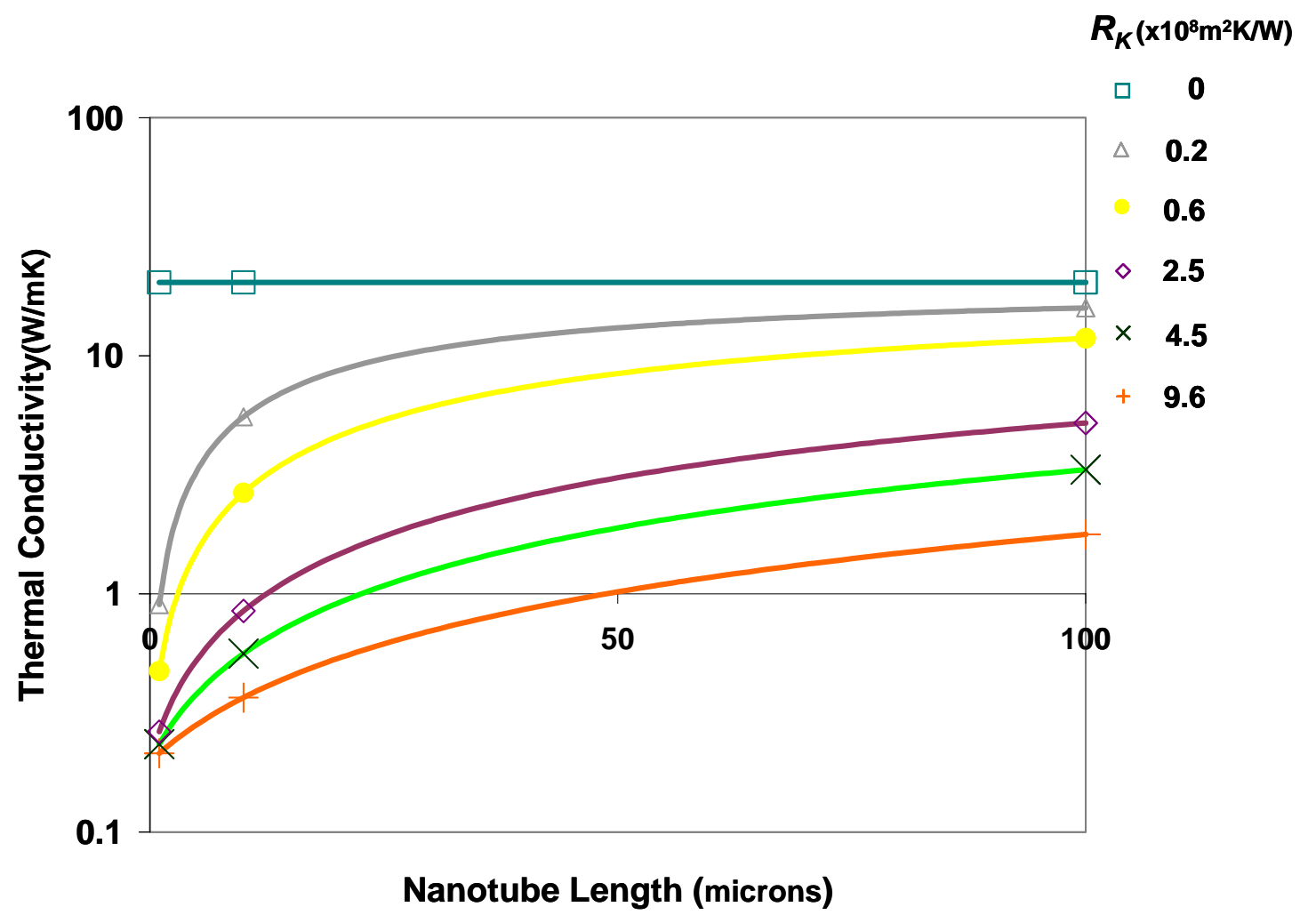

Fig. 6. The thermal conductivity of the nanocomposite as a function of nanotube length $(L)$ for a range of thermal interfacial resistance $R_{K}$ ) values. A value of $6000 \mathrm{~W} / \mathrm{mK}$ is used for the conductivity of the nanotube, $K_{c}$. The plotted points correspond to values of $L$ used in Figs. 5a-c. 


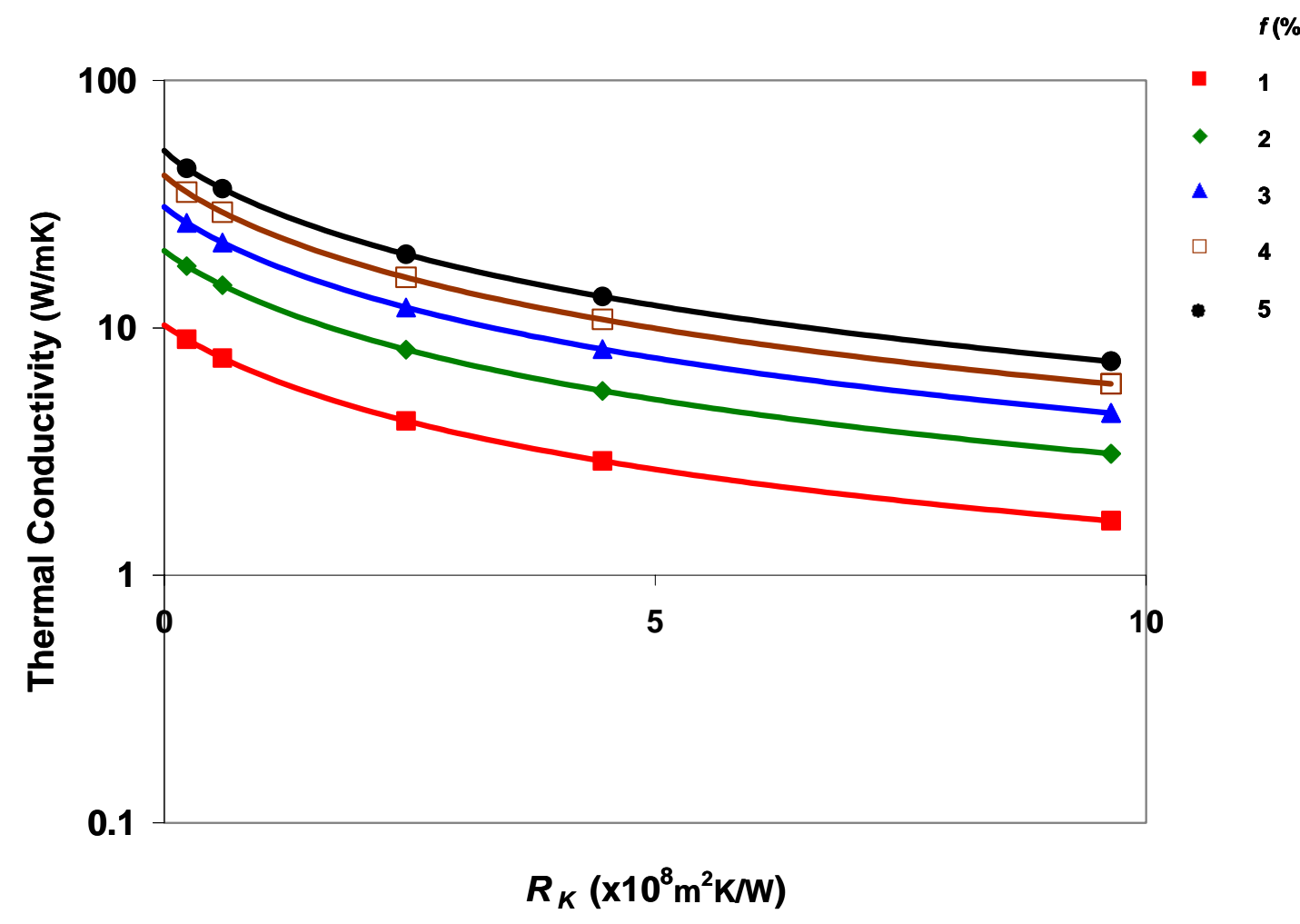

Fig. 7. The thermal conductivity of the nanocomposite as a function of interfacial thermal resistance $\left(R_{K}\right)$ for a range of volume fractions $(f=1-5 \%)$. A value of $10^{-4} \mathrm{~m}$ (100 microns) is used for the nanotube length, $L$. A value of $3000 \mathrm{~W} / \mathrm{mK}$ is used for the conductivity of the nanotube, $K_{c}$. The plotted points correspond to values obtained from the MD simulation with end-grafted chains of length $n=8$. 


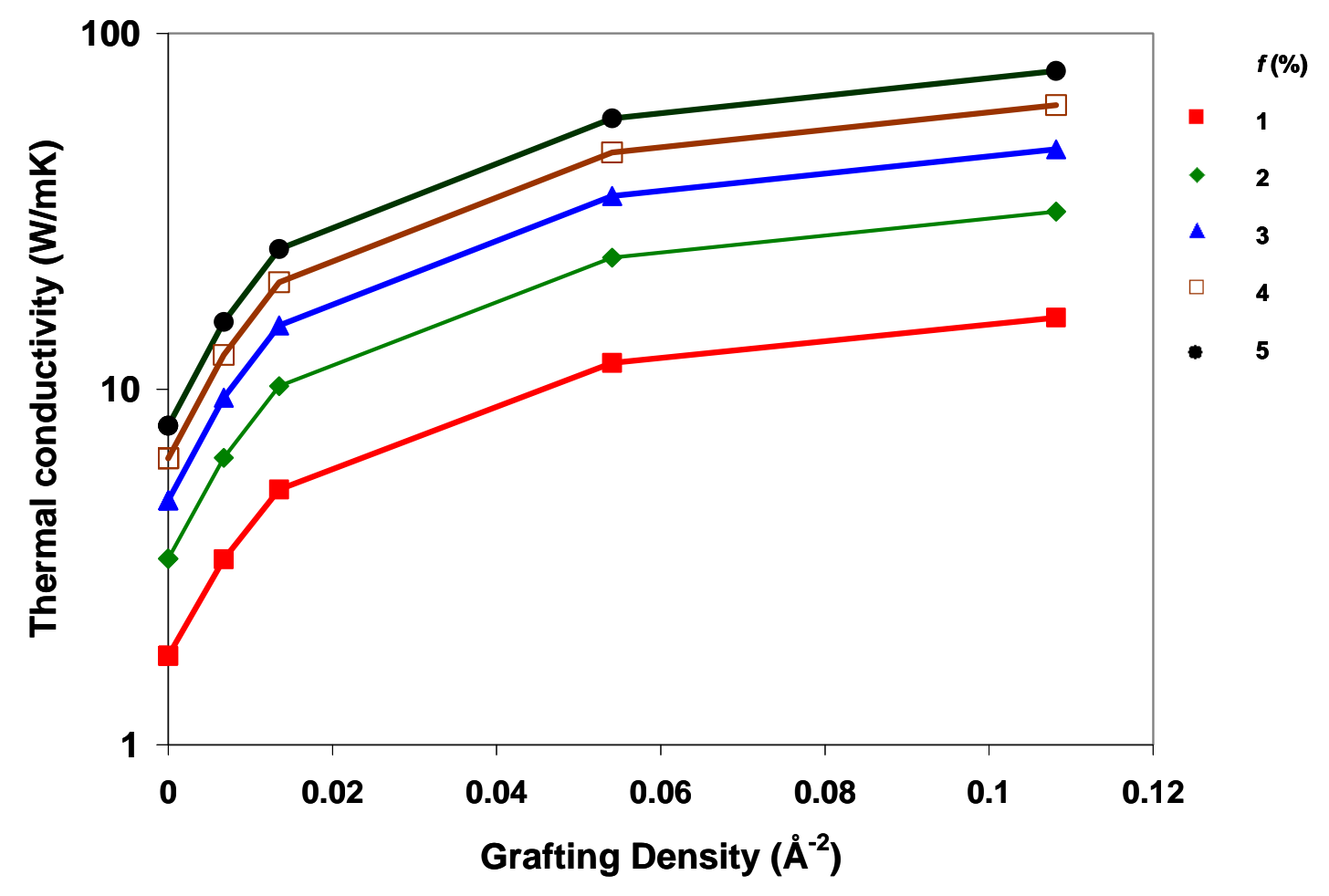

Fig. 8. The thermal conductivity of the composite to the matrix as a function of grafting density $(\sigma)$ for a range of volume fractions $(f=1-5 \%)$. A value of $10^{-4} \mathrm{~m}$ (100 microns) is used for the nanotube length, $L$. A value of $6000 \mathrm{~W} / \mathrm{mK}$ is used for the conductivity of the nanotube, $K_{c}$. The plotted points correspond to values obtained from the MD simulation with end-grafted chains of length $n=8$. 


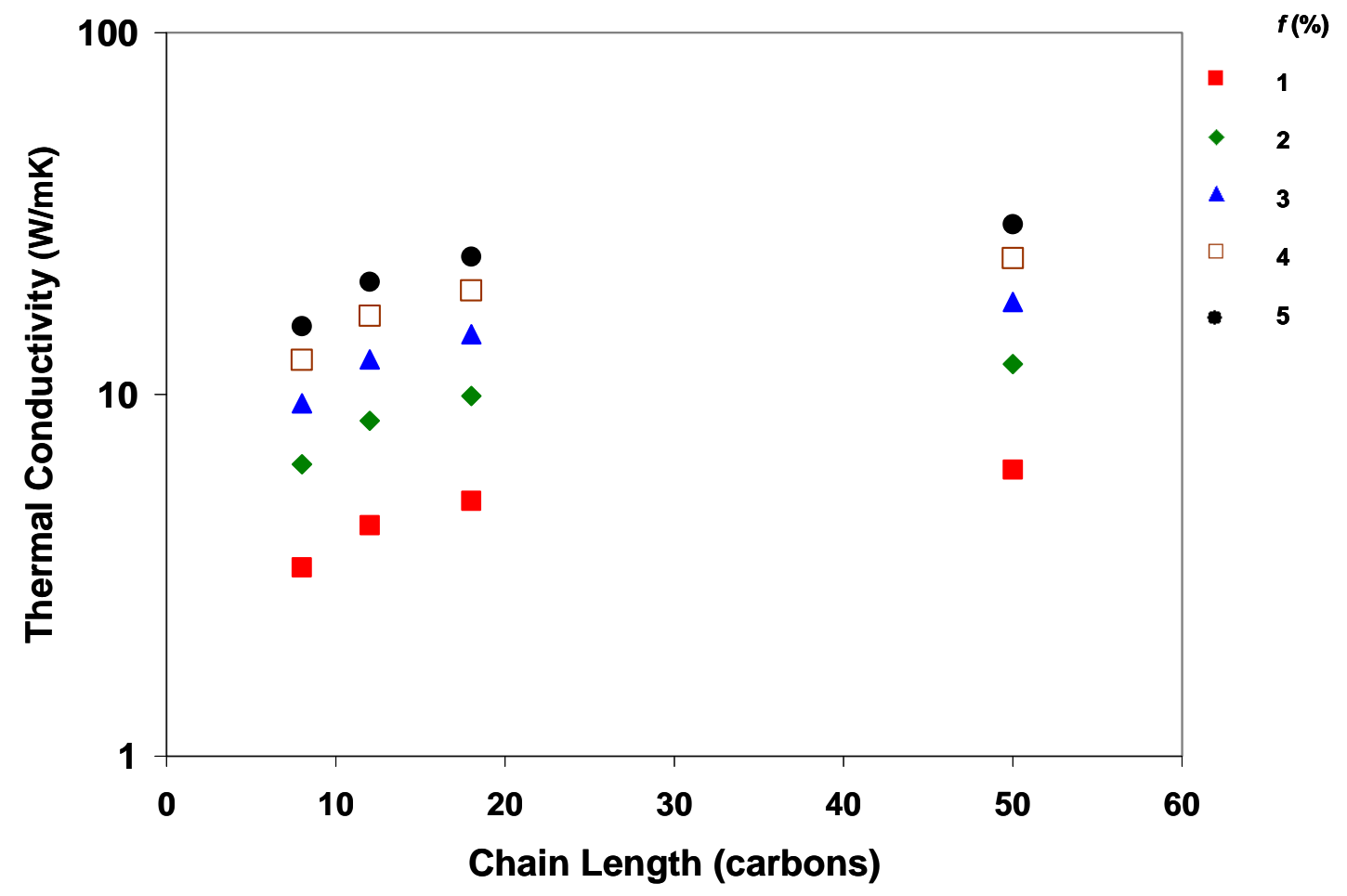

Fig. 9. The thermal conductivity of the nanocomposite as a function of length of the grafted chain for a range of volume fractions $(f=1-5 \%)$. A value of $10^{-4} \mathrm{~m}$ (100 microns) is used for the nanotube length, $L$. A value of $6000 \mathrm{~W} / \mathrm{mK}$ is used for the conductivity of the nanotube, $K_{c}$. 
